\title{
Riesgos en el consumo de bebidas azucaradas en estudiantes de la UAEH
}

\author{
Risks in the consumption of sugary drinks in students of the UAEH \\ Sergio A. Hernández-Islas ${ }^{a}$, Víctor H. Reyes-Lira ${ }^{a}$, Priscila Tapia-Bárcenas ${ }^{a}$, Iracema Islas- \\ Vegab $^{c}$, Miriam S. Hernández-Medina ${ }^{b}$, Zayda A. Trejo-Osti ${ }^{b}$, Ana L. López-González ${ }^{b}$, \\ Maricarmen Rivera Gómez ${ }^{c}$, Josefina Reynoso-Vázquez ${ }^{d}$, Jesús C. Ruvalcaba-Ledezma ${ }^{e^{*}}$
}

\begin{abstract}
:
The consumption of sweetened beverages today seems to represent daily life in terms of its intake and its effects associated with metabolic diseases such as; hypertension, insulin resistance, type 2 diabetes, obesity, overweight hypertriglyceridemia and metabolic syndrome. This causes important changes in the life of human populations to such a degree of being considered a serious public health problem, since it increases the incidence of cases of diseases that in turn cause greater expenditure in health care, the same situation that leaves glimpse the urgency of research being done in this field of knowledge.
\end{abstract}

Keywords:

Sugary drinks, soft drinks, risks, diseases.

\section{Resumen:}

El consumo de bebidas azucaradas hoy en día al parecer representa la cotidianidad en cuanto su ingesta y sus efectos asociados con enfermedades metabólicas tales como; hipertensión arterial, resistencia a la insulina, diabetes tipo 2, obesidad, sobrepeso hipertrigliceridemia y síndrome metabólico. Esto provoca cambios importantes en la vida de las poblaciones humanas a tal grado de ser considerado un grave problema de salud pública, ya que incrementa la incidencia de casos de enfermedades que a su vez provoca mayor gasto en atención de la salud, misma situación que deja entrever la urgencia de que se realice investigación en este campo del conocimiento.

Palabras Clave:

Bebidas azucaradas, refrescos, riesgos, enfermedades.

\section{Introducción}

El consumo masivo de refrescos (bebidas sin alcohol, carbonatadas y no carbonatadas que contienen edulcorantes calóricos y saborizantes) se consolidó desde la década de los años sesenta, al convertirse en parte de la dieta cotidiana, incluso en niños desde edades tempranas. Desde hace algunos años se ha observado que los países con mayor consumo anual por persona (litros) son Estados Unidos de Norteamérica (EUA) (200), México (150), Canadá (110) y Argentina (70). (1)

El consumo de bebidas calóricas en México es de los más elevados, con un incremento rápido en el consumo sin precedente en la historia mundial. Las consecuencias

\footnotetext{
${ }^{a}$ Instituto de Ciencias de la Salud, Universidad Autónoma del Estado de Hidalgo. priscilataba@ hotmail.com

b Instituto de Ciencias de la Salud, Universidad Autónoma del Estado de Hidalgo. iracema.islas.vega@gmail.com, miriamsel.ba8@gmail.com zayzui04@gmail.com, angie_0991@hotmail.com

${ }^{\mathrm{c}}$ Instituto de Ciencias de la Salud, Universidad Autónoma del Estado de Hidalgo.

${ }^{\mathrm{d}}$ Instituto de Ciencias de la Salud, Universidad Autónoma del Estado de Hidalgo.jreynosovazquez@hotmail.com

$e^{*}$ Autor de Correspondencia, Instituto de Ciencias de la Salud, Maestría en Salud Pública Universidad Autónoma del Estado de Hidalgo, https://orcid.org/0000-0002-5593-3946, Email: dcspjcarlos@gmail.com
} 
de ese nivel de ingesta se reflejan en los servicios de salud que atienden cada vez más pacientes con enfermedades metabólicas (2) como la hipertensión arterial, la resistencia a la insulina, diabetes tipo 2, obesidad abdominal, hipertrigliceridemia, síndrome metabólico, entre otras. (3)

La evidencia reciente sugiere una asociación entre la ingesta de refrescos azucarados y el riesgo de obesidad en los niños, pero los datos entre los adultos son limitados. Además de contribuir a la obesidad, los refrescos endulzados con azúcar pueden aumentar el riesgo de diabetes porque contienen grandes cantidades de jarabe de maíz con alto contenido de fructosa, lo que eleva la glucosa en sangre de manera similar a la sacarosa. (4) Además, en estudios aleatorizados en niños se ha observado que el consumo de bebidas azucaradas predice el IMC en los escolares y adolescentes en etapas posteriores a la vida. (5) Se ha especulado que los refrescos embotellados promueven la obesidad porque el consumo de energía obtenida desplaza la energía que proviene de los alimentos sólidos de la dieta. (6)

El aumento del IMC en niños se correlaciona con el desarrollo subsecuente de síndrome metabólico y, en consecuencia, con diabetes mellitus 2 y enfermedad cardiovascular. (7) En general, se ha observado que, en el país, entre la población de todas las edades y clases sociales, ha habido un consumo descontrolado de refrescos, y por lo regular de bebidas con alto valor energético; en lugar de beber agua natural, los mexicanos hemos optado por las bebidas azucaradas; tanto así que el gasto anual nacional en refrescos de cola supera al de los 10 alimentos básicos, y el consumo se inicia en el hogar desde etapas tempranas de la vida. (8) Muchas personas afirman que se sienten obligadas a comer alimentos dulces, similares en cierta forma a cómo un alcohólico puede sentirse obligado a beber. (9)

En este contexto, el gobierno mexicano implementó, a partir de enero de 2014, una política fiscal para reducir la carga de la obesidad y enfermedades relacionadas, que incluye, entre otras medidas, dos impuestos. El primero es un impuesto específico a bebidas azucaradas de un peso por litro a todas las bebidas no alcohólicas que contienen azúcar añadida -en polvo o en líquido listo para consumir-. El segundo es un impuesto advalorem de $8 \%$ a alimentos no básicos altos en densidad energética (AADE), definida como 275 kilocalorías por 100 gramos o más. (10)

La teoría del aprendizaje social establece que las conductas son adquiridas a partir de la imitación, y que los entornos de la familia, escolares, comunitarios, culturales y legislativos determinan las conductas humanas. (11) Por lo tanto, el propósito de este artículo es informar, que tan frecuentemente consumen este tipo de bebidas en estudiantes de la universidad y que clase de información tienen estos sujetos acerca de los riesgos en el consumo de bebidas de este tipo.

\section{Pregunta de investigación}

¿Cuáles son los riesgos en el consumo de bebidas azucaradas en estudiantes de medicina en ICSA?

\section{Objetivo General}

Determinar los riesgos en el consumo de bebidas azucaradas en estudiantes de medicina.

\section{Objetivos Específicos}

- Definir los diferentes tipos de bebidas azucaradas más consumidas en la comunidad estudiantil.

- Observar el grado de información que poseen azucaradas en la comunidad estudiantil.

- Definir los riesgos que ocasiona el consumo de bebidas azucaradas.

\section{Métodos}

En un estudio transversal-analítico se incluyeron 384 alumnos de distintos planteles universitarios que hay de la Universidad Autónoma del Estado de Hidalgo (ICSa, ICSHu, ICEA, ICBI, ICAP), de los cuáles 178 fueron hombres y 204 fueron mujeres.

Fueron seleccionados aleatoriamente, para responder la encuesta a través de los formularios de Google; primero en un iPad con conexión a internet recorriendo los distintos planteles y posteriormente a través de algunos contactos difundiendo la encuesta por la comunidad estudiantil de dichos planteles hasta que obtener el número de encuestados esperado. Para garantizar la transparencia de dicha encuesta, se modificó la configuración haciendo que cada usuario solo pudiera responder una sola vez por equipo electrónico.

En este estudio primero se interrogó sexo, su instituto de procedencia, sobre la frecuencia en su consumo de bebidas azucaradas, cuales consumen más, si conocían los riesgos que el consumo de estas provoca y si conocían los valores nutricionales que estas tienen. También si tenían familiares con diabetes que es una de 
las enfermedades provocadas a causa de estas bebidas (y que los haría propensos a esta).

\section{Resultados}

Se incluyeron 384 estudiantes de distintos planteles de la universidad autónoma del estado de Hidalgo; 179 hombres y 205 mujeres (Tabla 1), de distintas carreras. La primera pregunta fue" ¿A qué instituto perteneces" y como opciones de respuesta estaban disponibles "ICSa",
"ICSHu", "ICEA", "ICBI" y "otros"(haciendo referencia a los demás planteles que existen fuera de la ciudad de Pachuca y sus municipios aledaños. En este caso la mayoría de los encuestados fueron alumnos de ICSHu con 29.7\%(114 alumnos), seguido de ICSa con $27.1 \%$ (104 alumnos) después ICBI con un 21.9\% (84 estudiantes), ICEA con $17.4 \%$ (67 estudiantes) y finalmente otros institutos con 3.9\% (15 estudiantes). (Tabla2)

\begin{tabular}{|l|l|l|l|l|l|}
\hline \multicolumn{2}{|c|}{ Tabla 1. Género de estudiantes encuestados } \\
\hline \multirow{2}{*}{ Válidos } & Frecuencia & Porcentaje & $\begin{array}{l}\text { Porcentaje } \\
\text { válido }\end{array}$ & $\begin{array}{l}\text { Porcentaje } \\
\text { acumulado }\end{array}$ \\
\cline { 2 - 6 } & & & & \\
\cline { 2 - 6 } & $\mathrm{H}$ & 179 & 46.7 & 46.7 & 46.7 \\
\cline { 2 - 6 } & $\mathrm{M}$ & 205 & 53.3 & 53.3 & 100.0 \\
\cline { 2 - 6 } & Total & 384 & 100.0 & 100.0 & \\
\hline
\end{tabular}

\begin{tabular}{|c|c|c|c|c|c|}
\hline & & Frecuencia & Porcentaje & $\begin{array}{l}\text { Porcentaje } \\
\text { válido }\end{array}$ & $\begin{array}{l}\text { Porcentaje } \\
\text { acumulado }\end{array}$ \\
\hline \multirow[t]{6}{*}{ Válidos } & ICBI & 84 & 21.9 & 21.9 & 21.9 \\
\hline & ICEA & 67 & 17.4 & 17.4 & 39.3 \\
\hline & ICSa & 104 & 27.1 & 27.1 & 66.4 \\
\hline & $\mathrm{ICSHu}$ & 114 & 29.7 & 29.7 & 96.1 \\
\hline & Otro & 15 & 3.9 & 3.9 & 100.0 \\
\hline & Total & 384 & 100.0 & 100.0 & \\
\hline
\end{tabular}

Tabla 3. Frecuencia en el consumo de bebidas azucaradas

\begin{tabular}{|c|c|c|c|c|c|}
\hline & & Frecuencia & Porcentaje & $\begin{array}{l}\text { Porcentaje } \\
\text { válido }\end{array}$ & $\begin{array}{l}\text { Porcentaje } \\
\text { acumulado }\end{array}$ \\
\hline \multirow[t]{6}{*}{ Válidos } & Dos veces a la semana & 73 & 19.0 & 19.0 & 19.0 \\
\hline & Más de tres veces & 103 & 26.8 & 26.8 & 45.8 \\
\hline & $\begin{array}{l}\text { No consumo bebidas } \\
\text { azucaradas }\end{array}$ & 31 & 8.1 & 8.1 & 53.9 \\
\hline & Tres veces a la semana & 119 & 31.0 & 31.0 & 84.9 \\
\hline & Una vez a la semana & 58 & 15.1 & 15.1 & 100.0 \\
\hline & Total & 384 & 100.0 & 100.0 & \\
\hline
\end{tabular}

La segunda pregunta de nuestra encuesta fue: ¿Qué tan frecuentemente consumes bebidas azucaradas? (refresco, jugos, té helado, bebidas energéticas). Las opciones de respuesta fueron "no consumo bebidas azucaradas", "una vez a la semana", "dos veces a la semana", "tres veces a la semana" y "Más de tres veces a la semana".
La opción más respondida fue "Tres veces a la semana" con 31\%(119 alumnos), seguida de "más de tres veces" con 26.8\%(103 alumnos), "Dos veces a la semana" con 19\%(73 alumnos), "Una vez a la semana" con $15.1 \%$ (58 alumnos) y finalmente "no consumo bebidas azucaras" con $8.1 \%$ (31 personas). (Tabla 3)

La tercera pregunta fue ¿Qué bebidas azucaradas consumes más frecuentemente? Las opciones para 
respuesta fueron "Refrescos", "Té Helado", "Jugos", "Bebidas energéticas", "Otros".

La respuesta más repetida fue "Refrescos" con $29.9 \%$

(115 alumnos), seguida de "Jugos" con 29.4\% (113 alumnos), "Té helado" con 14.1\% (54 alumnos), "Bebidas energéticas" con 15.1\% (58 alumnos) y finalmente "otros" con 11.5\% (44 alumnos). (Tabla 4)

\begin{tabular}{|l|l|l|l|l|l|}
\hline \multicolumn{2}{|c|}{ Tabla 4. Bebidas azucaradas más consumidas } \\
\cline { 3 - 6 } & Frecuencia & Porcentaje & $\begin{array}{l}\text { Porcentaje } \\
\text { válido }\end{array}$ & $\begin{array}{l}\text { Porcentaje } \\
\text { acumulado }\end{array}$ \\
\hline \multirow{2}{*}{ Válidos } & & & 15.1 & 15.1 & 15.1 \\
\hline & Bebidas energéticas & 58 & 29.4 & 29.4 & 44.5 \\
\cline { 2 - 6 } & Jugos & 113 & 11.5 & 11.5 & 56.0 \\
\cline { 2 - 6 } & Otros & 44 & 29.9 & 29.9 & 85.9 \\
\cline { 2 - 6 } & Refrescos & 115 & 14.1 & 14.1 & 100.0 \\
\cline { 2 - 6 } & Té helado & 54 & 100.0 & 100.0 & \\
\cline { 2 - 6 } & Total & 384 & & \\
\hline
\end{tabular}

La cuarta pregunta fue ¿Conoces los riesgos en el consumo de este tipo de bebidas? Las opciones de "Si" obtuvo 51.3\%(197 alumnos) de las respuestas y respuesta fueron "si" y "no".

"No" 48.7\% (187 alumnos). (Tabla 5)

\begin{tabular}{|l|l|l|l|l|l|}
\hline \multicolumn{5}{|c|}{ Tabla 5. Conocimiento sobre los riesgos en el consumo de estas bebidas } \\
\hline \multirow{2}{*}{} & Frecuencia & Porcentaje & $\begin{array}{l}\text { Porcentaje } \\
\text { válido }\end{array}$ & $\begin{array}{l}\text { Porcentaje } \\
\text { acumulado }\end{array}$ \\
\hline \multirow{2}{*}{ Válidos } & No & 187 & 48.7 & 48.7 & 48.7 \\
\cline { 2 - 6 } & $\mathrm{Si}$ & 197 & 51.3 & 51.3 & 100.0 \\
\cline { 2 - 6 } & Total & 384 & 100.0 & 100.0 & \\
\hline
\end{tabular}

La quinta pregunta de la encuesta fue ¿Tienes familiares con diabetes? Las opciones de respuesta fueron "Si", "No" y "No lo sé".
La más respondida fue "Si" con 51.8\%(199 alumnos), seguida de "No" con 27.3\%(105 alumnos) y "No lo sé" con $20.8 \%$ (80 alumnos).(Tabla 6 )

\begin{tabular}{|l|l|l|l|l|l|}
\hline \multicolumn{5}{|l|}{ Tabla 6. Estudiantes con familiares diabéticos } \\
\hline \multirow{2}{*}{} & Frecuencia & Porcentaje & $\begin{array}{l}\text { Porcentaje } \\
\text { válido }\end{array}$ & $\begin{array}{l}\text { Porcentaje } \\
\text { acumulado }\end{array}$ \\
\hline \multirow{2}{*}{ Válidos } & No & 105 & 27.3 & 27.3 & 27.3 \\
\cline { 2 - 6 } & No lo sé & 80 & 20.8 & 20.8 & 48.2 \\
\cline { 2 - 6 } & Si & 199 & 51.8 & 51.8 & 100.0 \\
\cline { 2 - 6 } & Total & 384 & 100.0 & 100.0 & \\
\hline
\end{tabular}

La sexta pregunta fue ¿Conoces los valores nutricionales que poseen estas bebidas? Las opciones de respuesta fueron "Si" con 29.9\%(115 alumnos).y "No" con $70.1 \%(269$ alumnos).

\section{Discusión}

Se trató de un estudio realizado con un muestreo aleatorio realizado en la ciudad de Pachuca y el municipio de San Agustín Tlaxiaca donde se encuentran los planteles incorporados a la UAEH, enfocando esta encuesta en los estudiantes pertenecientes a esta. La mayoría de los estudiantes encuestados fueron mujeres con un $53.3 \%$ y el $46.7 \%$ fue correspondiente a los hombres. El instituto con mayor cantidad de alumnos encuestados fueron ICSHu con $29.7 \%$ cada uno, seguidos por ICSa con 27.1 , ICBI con $21.9 \%$, ICEA con $17.4 \%$ y otros con $3.9 \%$; estos resultados se dieron a causa de la manera en que se fue difundiendo la encuesta.

Como era esperado, la bebida azucarada más consumida por la comunidad universitaria fueron los 
refrescos con un 29.9\%, entre los que podemos destacar los de la familia Coca Cola que son los que se venden en las distintas cafeterías, Oxxo y máquinas expendedoras que se encuentran dentro de estos planteles. Después encontramos los jugos con un $29.4 \%$, Té helado con $14.1 \%$, bebidas energéticas con $15.1 \%$, y otros con $11.5 \%$. La mayoría de la comunidad estudiantil aseguró consumir estos productos más de tres veces a la semana, lo que refleja algo que ya se había señalado; que México es uno de los mayores consumidores de este tipo de bebidas en el mundo. (6)

La mayoría de las personas encuestadas aseguro que conocían los riesgos en el consumo de este tipo de bebidas, más sin embargo la diferencia con la cantidad de personas que desconoce los distintos riesgos es muy poca, y es algo que resulta contraproducente para la salud de la comunidad. Sólo el $51.3 \%$ conoce los riesgos que hay en el consumo de estas y el porcentaje restante podría resultar más expuesta a tener una vida menos saludable.

El $51.8 \%$ tiene familiares con Diabetes, el $27.3 \%$ no los tiene y el $20.8 \%$ desconoce si los tiene. Al ser familiar de alguna persona con diabetes, es de suma importancia que personas que tienen familiares con Diabetes asocien sus distintos hábitos y con los distintos alimentos que consumen día a día. Así como también se debería tener conocimiento en todos los casos sobre si se tienen o no familiares con este padecimiento.

Finalmente, al encuestar a esta muestra de estudiantes sobre si conocían los valores nutricionales de este tipo de bebidas, obtuvimos resultados negativos. El $70.1 \%$ de la población desconoce los valores de lo que están consumiendo; por lo que no se lleva un correcto control calórico.

\section{Conclusiones}

El consumo de bebidas energéticas en México es de los más elevados en el mundo, y su aumento no tiene precedente en la historia mundial de acuerdo con los datos del comité de expertos para las recomendaciones de bebidas para la población mexicana. (6)
Entre los estudiantes el consumo de bebidas azucaradas es muy elevado; por eso se requiere de distintas estrategias para buscar disminuir las cifras que se vieron reflejadas en este muestreo. Asimismo, se debe limitar de alguna manera la venta de estos productos dentro de las instalaciones, pues este tipo de bebidas son las que se consumen en mayor medida por los estudiantes universitarios.

\section{Conflicto de intereses}

Los autores declaramos que no existe conflicto de intereses para la publicación del presente artículo.

\section{Referencias}

[1] Pediatría, Comité de Nutrición de la Asociación Española de Pediatria. Consumo de zumo de frutas y bebidas refrescantes por niños y adolescentes en España, implicaciones para la salud de su mal uso y abuso. An Pediatr, 2003, Vol. 58. 584-593.

[2] María del Rosario Rodríguez-Burelo, María Isabel Avalos-García, Concepción López-Ramón. Consumo de bebidas de alto contenido calórico en México: un. s.l. : SALUD EN TABASCO, Vol. 20.

[3] Schulze MB, Liu S, Rimm EB, Manson JE, Willett WC, Hu FB. Glycemic index, glycemic load, and dietary fiber intake and incidence of type 2 diabetes in younger and middle-aged women. Am J Clin Nutr, 2004, Vol. 80: 348-356.

[4] Schulze M., Manson J., Ludwig D., Colditz G., Stampfer M., Willett W., Hu F. Sugar-Sweetened Beverages, Weight Gain, and Incidence of Type 2 Diabetes in Young and Middle-Aged Women. JAMA, 2004, Vol. 8: 927-934.

[5] Perez-Morales E, Bacardí-Gascón M, Jiménez-Cruz A. Su-garsweetened beverage intake before 6 years of age and weight or BMI status among older children; systematic review of pros-pective studies. Nutr Hosp, 2003, Vol. (28): 47-51.

[6] Johnson RK, Frary C. Choose beverage and foods to moderate your intake of sugars: The 2000 dietary guidelines for Americans, What's all the fuss about? J Nutr, 2001, Vol 131: 276-277.

[7] Frenk B. P. y Márquez E. Diabetes mellitus tipo 2 en niños y adolescentes. Med Int Mex, 2010, Vol. 1. 36-47.

[8] Rivera JA, Muñoz-Hernández O, Rosas-Peralta M, Aguilar-Salinas CA, Popkin BM, Willet WC. Consumo de bebidas para una vida saludable: recomendaciones para la población mexicana. Salud Pública Mex, 2008, Vol. 50: 173- 195.

[9] Avena N., Rada P., Hoebel B. Evidence for sugar addiction: Behavioral and neurochemical effects of intermittent, excessive sugar intake. Neurosci Biobehav Rev. 2008, Vol. 1. 20-39.

[10] Colchero, M Arantxa, y otros. Cambios en los precios de bebidas. 2, s.l. Salud Pública de México, 2017, Vol. 59.

[11] Ramírez, G. Díaz, y otros. Efecto de la publicidad de alimentos anunciados en la televisión sobre la preferencia y el consumo de alimentos: revisión sistemática. Nutr. Hosp. 2011, 6, s.1. : Vol. 26. 